\title{
SCIENTIFIC REPORTS

\section{OPEN Vaginal microbiota diverges in sows with low and high reproductive performance after porcine reproductive and respiratory syndrome vaccination}

\author{
L. P. Sanglard ${ }^{1}$, S. Schmitz-Esser ${ }^{1,2}$, K. A. Gray ${ }^{3}$, D. C. L. Linhares ${ }^{4}$, C. J. Yeoman ${ }^{5}$, \\ J. C. M. Dekkers ${ }^{1}$, M. C. Niederwerder ${ }^{6} \&$ N. V. L. Serão ${ }^{1 *}$
}

Previous studies have demonstrated evidence for a relationship between the vaginal microbiome and reproductive performance, suggesting the vaginal microbiota may serve as a tool to predict farrowing outcomes in commercial pigs. In this study, we compared the vaginal microbiome in sows with low and high farrowing performance and used it to classify animals with contrasting reproductive outcomes in commercial sows following immune challenge with porcine respiratory and reproductive syndrome (PRRS) vaccination. Eighteen microbes were differentially abundant ( $q$-value $<0.05$ ) between the Low and High farrowing performance groups. Among them, Campylobacter, Bacteroides, Porphyromonas, Lachnospiraceae unclassified, Prevotella, and Phascolarctobacterium were also selected in the discriminant and linear regression analyses, and could be used as potential biomarkers for reproductive outcomes. The correct classification rate in the two groups was $100 \%$. In conclusion, this study demonstrates that vaginal microbiota collected after PRRS vaccination could be potentially used to classify sows into having low or high farrowing performance in commercial herds.

Farrowing performance in commercial sows is a key component of cost-effectiveness in the swine industry. Reproductive technologies (e.g., artificial insemination) have allowed producers to maximize the use of existing resources in the production systems to improve reproductive efficiency ${ }^{1}$. Strategies such as selection have allowed the genetic improvement of sows to exhibit enhanced farrowing performance ${ }^{2}$. However, variation in these traits is little explained by the individual's genetic, creating challenges for rapid improvements in farrowing performance. Recently, it has been shown that host-associated microbiota plays a role in shaping phenotypes of humans and animals ${ }^{3}$. For example, the vaginal microbiota has been shown to impact preterm birth and neonatal health ${ }^{4}$. In beef cattle, the vaginal microbiota was used to distinguish between heifers that were able to establish pregnancy from those that were not ${ }^{2}$, suggesting its potential to be used to identify animals with favorable reproductive performance. In addition, relationships between the microbiota and immune response have been previously observed $^{5}$. Therefore, microbiota collected after modified live virus (MVL) porcine reproductive and respiratory syndrome (PRRS) vaccination may be an alternative indicator of reproductive performance in commercial sows. In addition, the microbiota is relatively easy to collect and can be profiled with considerable ease using current molecular techniques ${ }^{6}$, making it a potential candidate phenotype to predict reproductive outcomes in commercial sows. Therefore, the objectives of this study were to identify differences in composition and alpha diversity of vaginal microbiota between sows with low and high farrowing performance, and to use the vaginal microbiota to classify animals with contrasting reproductive outcomes following PRRS vaccination.

${ }^{1}$ Department of Animal Science, lowa State University, Ames, 50011, USA. ${ }^{2}$ Interdepartmental Microbiology Graduate Program, lowa State University, Ames, 50011, USA. ${ }^{3}$ Smithfield Premium Genetic, Rose Hill, 28458, USA. ${ }^{4}$ Department of Veterinary Diagnostic \& Production Animal Medicine, lowa State University, Ames, 50011, USA. ${ }^{5}$ Department of Animal \& Range Sciences, Montana State University, Bozeman, 59717, USA. ${ }^{6}$ Department of Diagnostic Medicine/Pathobiology, Kansas State University, Manhattan, 66506, USA. *email: serao@iastate.edu 
a

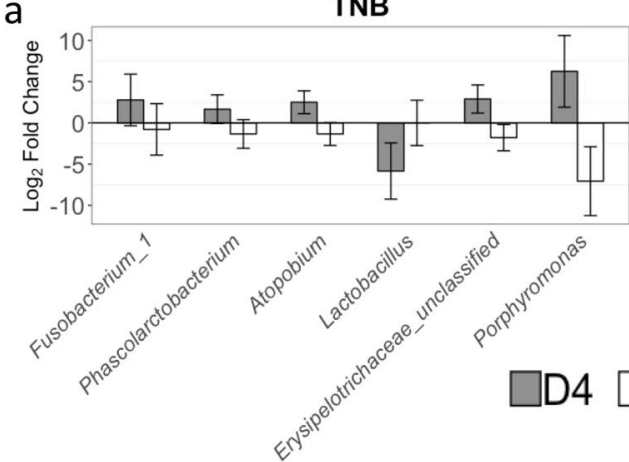

C

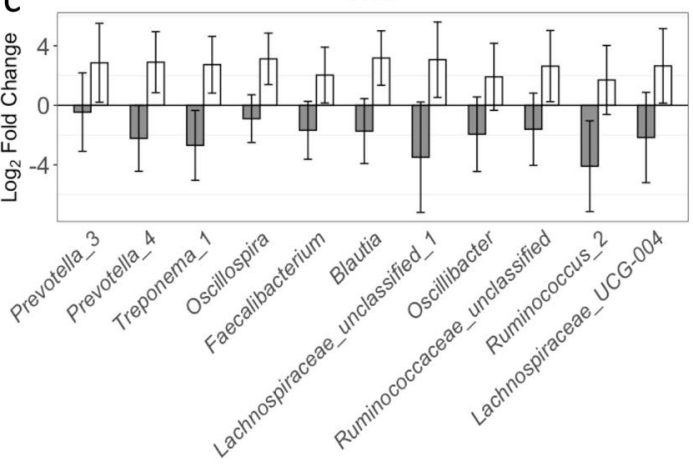

e

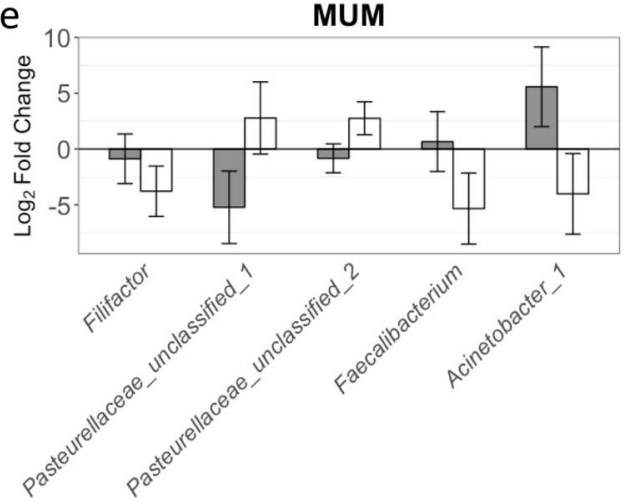

b

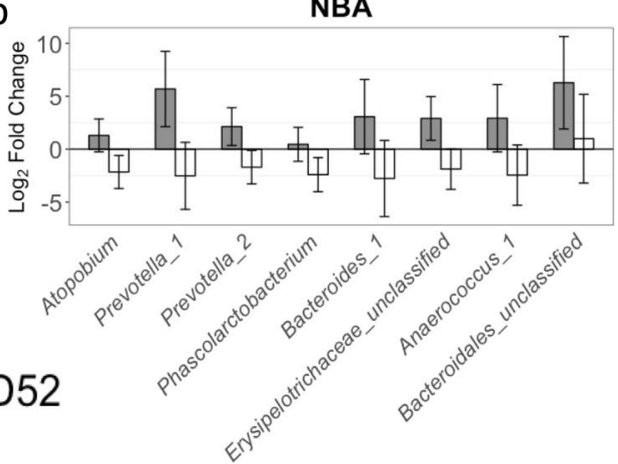

NBD

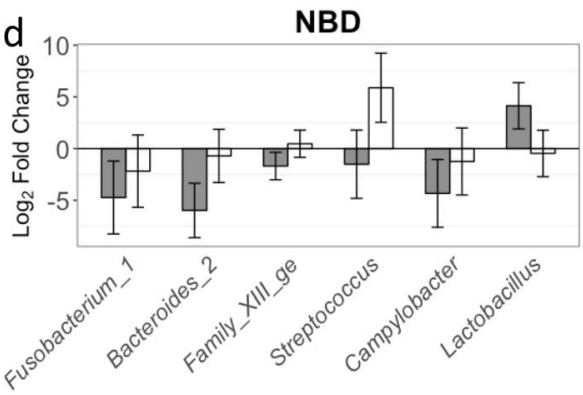

Figure 1. Differentially abundant microbes for the interaction between farrowing performance group and day of microbiome collection. The groups were defined based on the best/worst performance of (a) total number born, TNB; (b) number born alive, NBA; (c) number of weaning, NW; (d) number born dead, NBD; (e) number of piglets mummified, MUM; and (f) pre-weaning mortality, PWM. Positive and negative values represent higher abundance in the groups with high and low farrowing performance, respectively. Bar colors represent collections of days 4 (D4; gray) and 52 (D52; white) after vaccination to porcine respiratory and reproductive syndrome. The errors bars correspond to $95 \%$ confidence interval.

\section{Results}

Identification of DAMs and alpha diversity between sows with Low and High farrowing performance. Differences in microbiota $(q$-value $<0.05)$ relative abundances were identified for the interaction between farrowing performance group and day of collection (Fig. 1) and for the main effect of farrowing performance group (Fig. 2).

For the interaction, differentially abundant microbes (DAMs) were identified for total number born (TNB), number born alive (NBA), number of piglets weaning (NW), number born dead (NBD), number of piglets mummified (MUM), and pre-weaning mortality (PWM; Fig. 1). For TNB (Fig. 1a), Fusobacterium_1, Phascolactobacterium, Atopobium, Eryspelotrichaceae, and Pophyromonas had higher abundance in the High farrowing performance group compared to the Low with the $\log _{2}$ fold change $\left(\log _{2} \mathrm{FC}\right)$ being larger on day 4 (D4) compared to day 52 (D52). In contrast, Lactobacillus had lower abundance in the High farrowing group compared to the Low farrowing group on D4 but did not differ on D52. For NBA (Fig. 1b), the identified DAMs Atopobium, Bacteroides_1, Phascorlactobacterium, Bacteroidales_unclassified, Erysipelotrichaceae_unclassified, Anaerococcus_1, Prevotella_1, and Prevotella_2 had higher abundance in the High group compared to the Low 

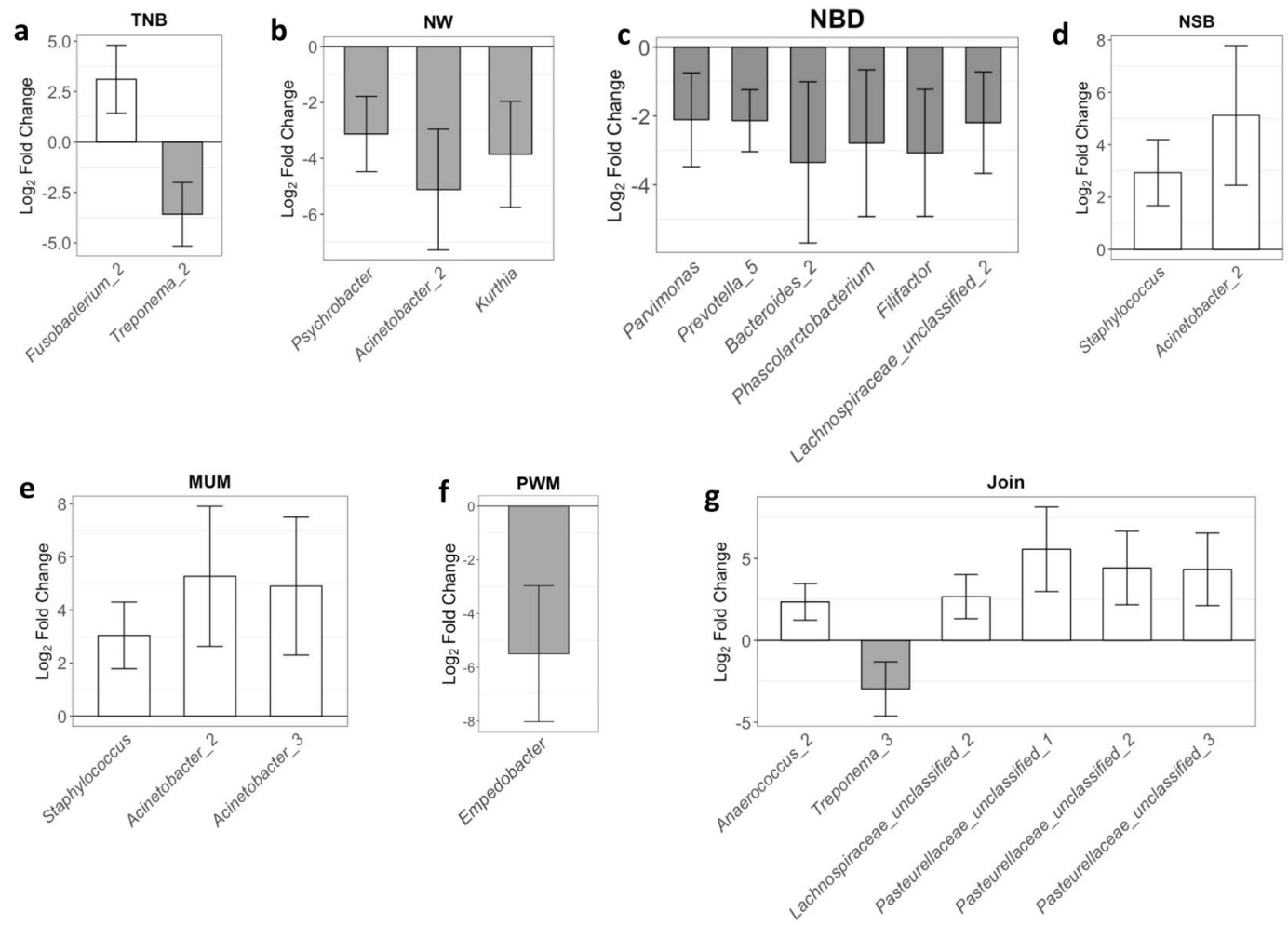

Figure 2. Differentially abundant microbes (DAMs) between groups of animals classified as High or Low farrowing performance. (a) Total number born, TNB; (b) number weaning, NW; (c) number born dead, NBD; (d) number of stillborn, NSB; (e) number of piglets mummified, MUM; (f) pre-weaning mortality; and (g) NBA associated with NBD, Join. The errors bars correspond to $95 \%$ confidence interval. The colors represent greater (white) or lower (gray) abundance in High performance group compared to the Low performance group.

group on D4 compared to D52, when the abundance in the Low group compared to the High group increased. For NW (Fig. 1c), Prevotella_3, Prevotella_4, Treponema_1, Oscillospira, Faecalibacterium, Blautia, Lachnospiraceae_ unclassified_1, Oscillibacter, Ruminococcus, and Lachnospiraceae_UCG-004 had lower abundance in the High group compared to the Low group on D4. They had higher abundance in the High group on D52. For NBD (Fig. 1d), Fusobacterium_1, Bacteroides_2, and Campylobacter had lower abundance in the High group compared to the Low group on both D4 and D52, but the difference in abundance decreased from D4 to D52. There was lower abundance of Family XIII and Streptococcus in the High group compared to the Low group on D4 compared to D52, when the abundance in the High group compared to the Low group increased. Finally, Lactobacillus was more abundant in the High group compared to the Low group on D4 compared to D52, when the abundance in the High group compared to the Low group decreased. For MUM (Fig. 1e), Pasteurellaceae_unclassified_1 and Pasteurellaceae_unclassified_2 had greater abundance in the Low compared to the High group on D4 and on D52 the abundance in the High group compared to the Low group increased. Filifactor had lower abundance in the High group compared to the Low group on D52 and on D4. Faecalibacterium and Acinetobacter_1 had higher abundance in the High group compared to the Low group on D4 compared to on D52, when the abundance in the Low group compared to the High group increased. For PWM (Fig. 1f), Campylobacter had higher abundance on High group on D4 but not on D52 and Veillonellaceae unclassified had higher abundance on Low group on D4 and the opposite on D52.

For the main effect of farrowing performance group, DAMs were identified $(q$-value $<0.05)$ for TNB, NW, NBD, number of stillborn piglets (NSB), MUM, PWM, and Join (representing joint analysis of NBA and NBD), as depicted in Fig. 2. Positive and negative $\log _{2} \mathrm{FCs}$ refer to higher and lower abundance on High compared to the Low farrowing performance groups, respectively. For TNB (Fig. 2a), 2 DAMs were identified: Fusobacterium_2 $\left(\log _{2} \mathrm{FC}=3.11\right)$ and Treponema_2 $\left(\log _{2} \mathrm{FC}=-3.57\right)$. For NW (Fig. $\left.2 \mathrm{~b}\right)$, Psychrobacter $\left(\log _{2} \mathrm{FC}=-3.13\right)$, Acinetobacter_2 $\left(\log _{2} \mathrm{FC}=-5.12\right)$, and Kurthia $\left(\log _{2} \mathrm{FC}=-3.85\right)$ showed greater abundance in the Low performance group compared to the High. Six DAMs between sows from High and Low groups were identified when the groups were defined based on NBD (Fig. 2c). For NBD, all DAMs were more abundant in the Low group compared to the High group: Parvimonas $\left(\log _{2} \mathrm{FC}=-2.11\right)$, Prevotella_5 $\left(\log _{2} \mathrm{FC}=-2.13\right)$, Bacteroides_2 $\left(\log _{2} \mathrm{FC}=-3.35\right)$, Phascolarctobacterium $\left(\log _{2} \mathrm{FC}=-2.79\right)$, Filifactor $\left(\log _{2} \mathrm{FC}=-3.07\right)$, and Lachnospiraceae unclassified_2 $\left(\log _{2} \mathrm{FC}=-2.19\right)$. For NSB (Fig. 2d) and MUM (Fig. 2e), Staphylococcus and Acinetobacter_2 were more abundant in the High group, with $\log _{2} \mathrm{FC}$ of 2.93 and 5.11 for NSB, respectively, and 3.04 and 4.89 (Acinetobacter_2) and 5.26 (Acinetobacter_1) for MUM, respectively. For PWM (Fig. 2f), Empedobacter $\left(\log _{2} \mathrm{FC}=-5.49\right)$ was more abundant on the Low group compared to the High. For Join (Fig. $2 \mathrm{~g}$ ), the DAMs were represented by Anaerococcus_2 $\left(\log _{2} \mathrm{FC}=2.35\right)$, Treponema_3 $\left(\log _{2} \mathrm{FC}=-2.97\right)$, Lachnospiraceae_unclassified_2 

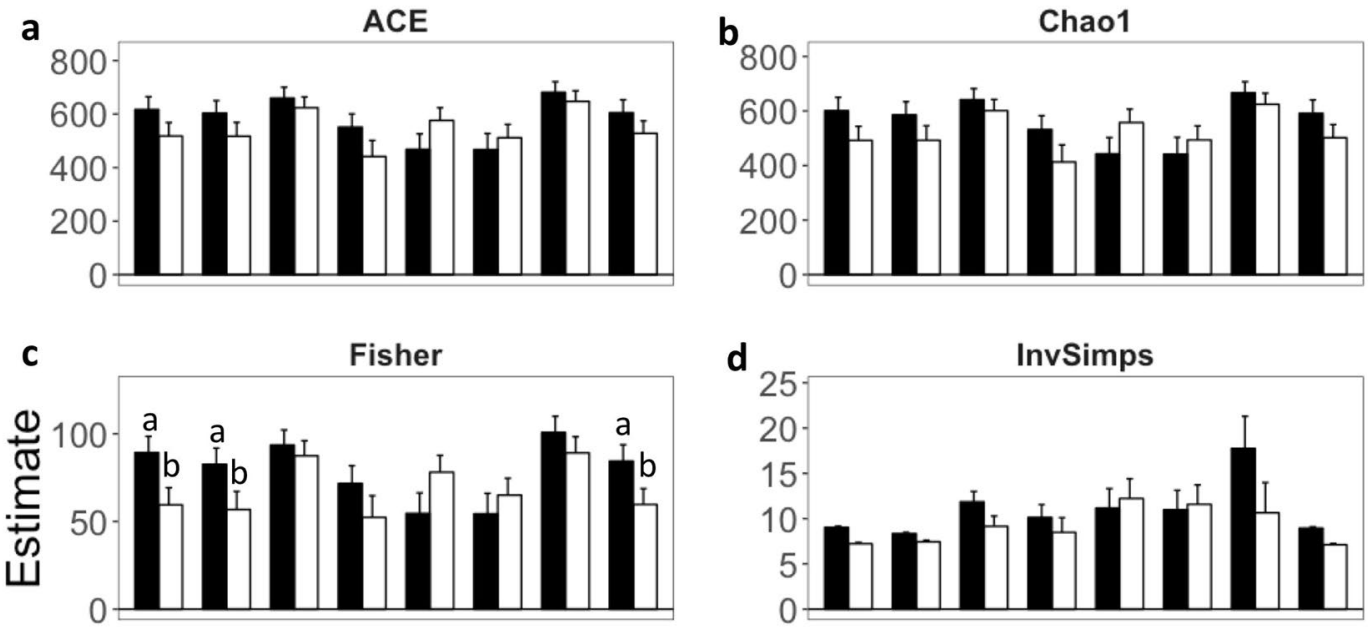

e

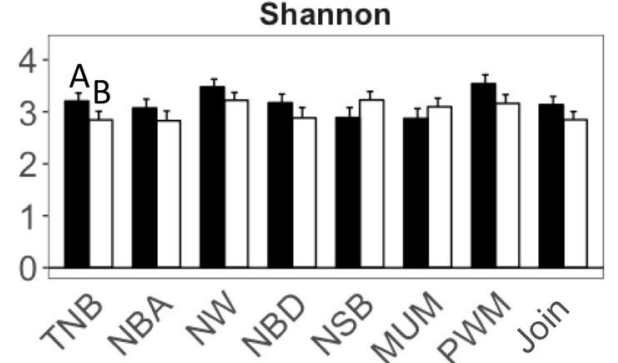

f

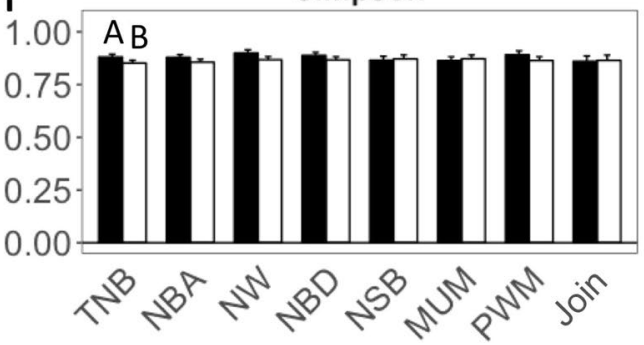

Low performance $\square$ High performance

Figure 3. Alpha-diversity measurements for Low and High farrowing performance groups. The alpha-diversity measurements are (a) abundance-based coverage estimator (ACE), (b) Chao1, (c) Fisher, (d) Inverse Simpson (InvSimps), (e) Shannon, and (f) Simpson. Farrowing performance groups were defined based on the best/worst performance of total number born (TNB), number born alive (NBA), number weaned (NW), number born dead (NBD), number of stillborn (NSB), number of piglets mummified (MUM), pre-weaning mortality (PWM), and combination of NBA and NBD (Join). The errors bars correspond to $95 \%$ confidence interval. Means lacking common lower- and uppercase letters are statistically different at $P<0.05$ and $<0.10$, respectively.

$\left(\log _{2} \mathrm{FC}=2.66\right)$, Pasteurellaceae_unclassified_1 $\left(\log _{2} \mathrm{FC}=5.56\right)$, Pasteurellaceae_unclassified_2 $\left(\log _{2} \mathrm{FC}=4.34\right)$, and Pasteurellaceae_unclassified_3 $\left(\log _{2} \mathrm{FC}=4.32\right)$, being Treponema_3 the only one more abundant in the Low compared to the High group. No DAMs $(q$-value $>0.05)$ were identified for NBA. For the effect of day, 180, $250,106,8,60,41,96$, and 179 DAMs were identified ( $q$-value $<0.05$ ) for TNB, NBA, NW, NBD, NSB, MUM, PWM, and Join, respectively (Supplementary Table S1). The operational taxonomic units (OTUs) that were differentially abundant in most analyses performed ( 5 out of 8 ) were Anaerococcus, with higher abundance on D4 compared to D52 (range $\log _{2} \mathrm{FC}=1.30$ to 5.16); Ruminococcaceae, with higher abundance on D52 compared to D4 (range $\log _{2} \mathrm{FC}=-0.81$ to -5.48 ); Clostridium sensu stricto, with higher abundance on D52 compared to D4 (range $\log _{2} \mathrm{FC}=-0.79$ to -4.56 ); Lachnospiraceae unclassified, with higher abundance on D52 compared to D4 (range $\log _{2} \mathrm{FC}=-1.39$ to -5.29 ); and Prevotellaceae, with higher abundance on D52 compared to D4 (range $\log _{2} \mathrm{FC}=-1.16$ to -4.11$)$.

Differences in the microbiota alpha-diversity between farrowing performance groups are presented on Fig. 3. Differences were only observed in Join, NBA, and TNB $(P$-value $<0.05)$ for Fisher alpha-diversity. There was a higher Fisher alpha-diversity in the Low performance group compared to the High performance. For TNB, there was also a tendency $(P$-value $<0.10)$ for higher alpha-diversity based on Shannon and Simpson measurements in the Low performance group compared to the High group.

Classification of gilts into farrowing performance groups based on OTU abundance. The selected OTUs, along with their standardized coefficients, are shown in Supplementary Table S2. The number of selected $(P$-value $<0.05)$ OTUs ranged from 14 to 16 . The OTUs classified as Lachnospiraceae unclassified and Ruminococcaceae were selected in the analyses based on all traits. Porphyromonas, Campylobacter, Fillifactor, Prevotella, Actinobacillus, and Fusobacterium were selected in at least 3 analyses. All linear discriminant analyses (LDA) had $R^{2}>0.99$ and $P$-value $<0.001$. The leave-one-out cross-validation resulted in a correct classification rate of $100 \%$ for all analyses. 
a
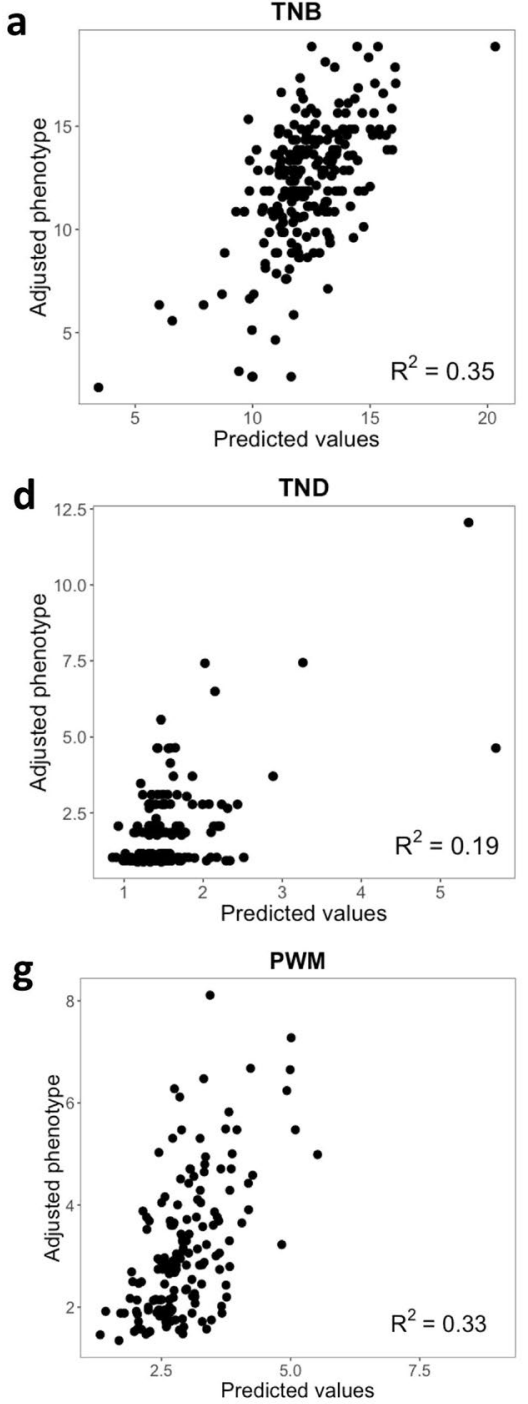
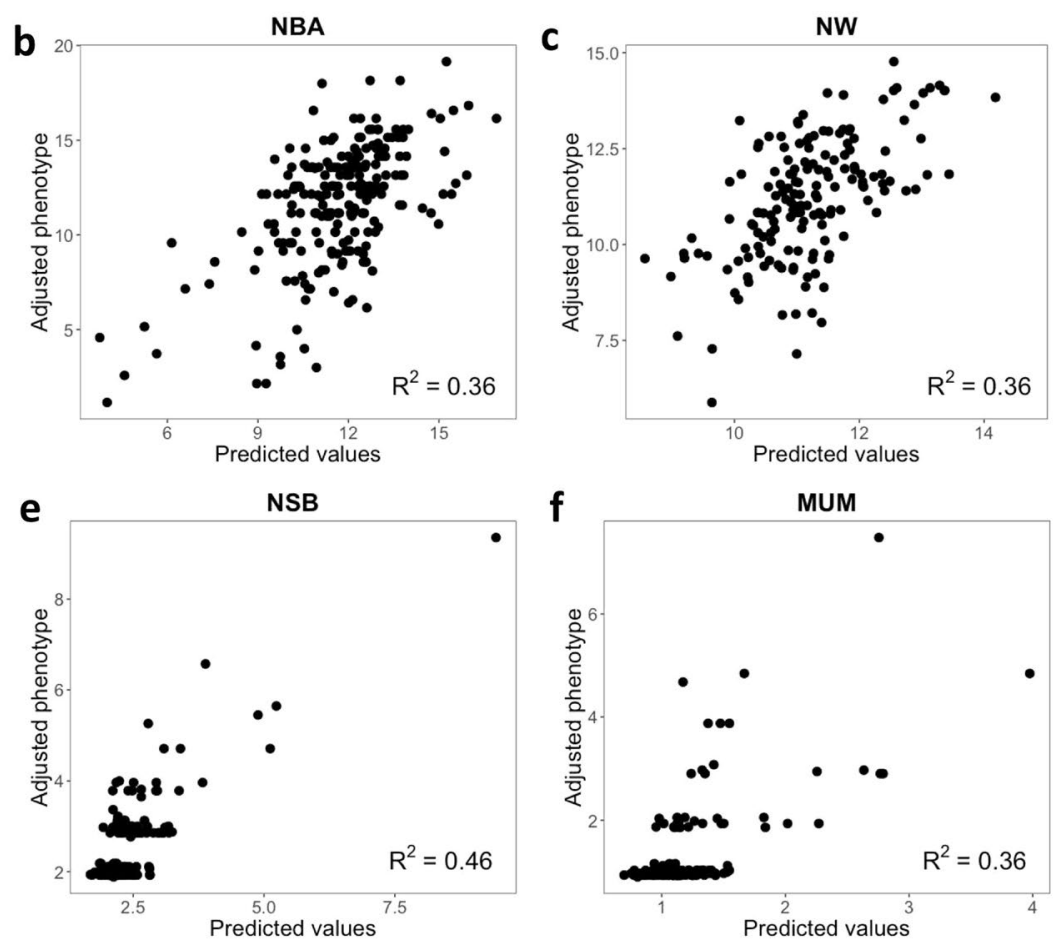

Figure 4. Stepwise linear regression of operational taxonomic units (OTUs) on farrowing performance traits: (a) total number born, TNB; (b) number born alive, NBA; (c) number weaning, NW; (d) number born dead, NBD; (e) number of stillborn, NSB; (f) number of piglets mummified, MUM; and (g) pre-weaning mortality, PWM. The $\mathrm{x}$-axis represents the predicted values and the $\mathrm{y}$-axis represents the adjusted phenotype. R-squares correspond to the coefficient of determination $\left(R^{2}\right)$.

The LDA was also performed for each day separately and the selected OTUs along with their standardized coefficients can be seen on Supplementary Table S3. For this, the leave-one-out cross-validation resulted in a correct classification rate of $100 \%$ for all analyses, with exception of NBA and MUM on D4, and NW on D52, which had a correct classification rate of $95 \%$ (i.e., only one sample was misclassified). In these cases, the accuracies of the classification [i.e., area under the receiver operating characteristic (ROC) curve; AUC] were 0.95, 0.89, and 0.95, respectively, as can be observed on Supplementary Fig. S1.

Prediction of farrowing performance based on OTU. The stepwise linear regression (Fig. 4; Supplementary Table S4) reveled that a moderate linear relationship between reproductive traits and vaginal microbiota, with $R^{2}$ ranging from 0.19 (NBD) to 0.46 (NSB). The number of OTUs selected $(P$-value $<0.15)$ ranged from 4 (NBD) to 16 (NSB) across both days and the most frequently included OTUs were Lachnospiraceae, Ruminococcaceae, Ruminiclostridium, Subdoligranulum, and Alloprevotella, which were selected for 6, 5, 2, 3, and 2 analyses, respectively. These analyses were also performed for each day separately and can be seen on Supplementary Table S5 and Supplementary Fig. S2.

\section{Discussion}

Previous studies have shown that antibody response to PRRS virus following PRRS infection is associated with reproductive outcomes during natural PRRS infection ${ }^{7,8}$. In addition, a relationship between the microbiota and immune response has been observed ${ }^{5}$. We, therefore, hypothesized that microbiota collected after MLV PRRS vaccination may be an alternative indicator of reproductive performance in commercial sows. In addition, the 
vaginal microbiota has been shown to be associated to reproductive outcomes in humans and cattle ${ }^{4}$ and has the advantage of being relatively easy to collect and possible to be collected at early ages. Therefore, in this work, we assessed and corroborated the possibility of using vaginal microbiota to identify animals with contrasting reproductive performance in commercial sows.

The vaginal microbiota was collected at 4 and 52 days following the MLV PRRS vaccination. The development of immune response to PRRS occurs late, with production of neutralizing antibodies peaking at 9 to 11 weeks (Lopez \& Osorio) $^{9}$. However, initial response can be observed as early as 2 days post infection with the induction of interleukin-10 (IL-10) associated with the generation regulatory T cells. In addition, PRRS outbreaks can be identified within a few days. Thus, by investigating the microbiota on days 4 and 52 after vaccination, we focused on collection of data soon after a PRRS MLV challenge has occurred (day 4), as well during peak antibody response $(\text { day } 52)^{10}$. In this study, we had no intention to assess the effect of vaccination on the microbiota, which would have required evaluation of a control group on non-vaccinated gilts; however, we recognize that, given the relationship between the microbiota and immune respons ${ }^{11}$, the infection with PRRS virus through vaccination during period of collection may be playing a role in the modulation of the vaginal microbiota. It should be noted that the interactions identified between days of collection and farrowing performance group on OTU abundance may be the result of modulation of the immune response on the microbiota, together with the effect of time and age.

The microbiota has been associated with several phenotypic outcomes (i.e., diseases, stress, reproduction); however, the mechanisms involved in the interaction between the host and the microbiota is still unclear. One possible mechanism that relates the microbiota to reproduction appears to involve hormones ${ }^{12}$. Indeed, the gut microbiota can produce and secrete hormones, while hormones can stimulate or inhibit the development of specific microbes, characterizing a bidirectional relationship ${ }^{12}$. Previous studies of the gut microbiota in mice have found correlations of some of the microbes identified in this study, such as Lachnospiraceae, Clostridium, Prevotella, and Ruminococcaceae, with circulating hormones (i.e., leptin and urinary estrogen; ${ }^{13}$ ). Also, Oscillibacter has been associated with higher levels of glucocorticoid in the gut microbiota of gorillas ${ }^{14}$. Thus, exploring the host microbiota and relating it to phenotypes of interest could help the swine industry improve animal reproductive performance.

A total of 50 unique DAMs were identified across all analyses. Of these, Phascolarctobacterium, Filifactor, Atopobium, Campylobacter, Staphylococcus, Treponema, Erysipelotrichaceae unclassified, Acinetobacter, and Faecalibacterium were identified in multiple analyses. Phascolarctobacterium, which was identified in the analyses based on TNB, NBA, and NBD, was more abundant in the Low performance group. Similarly, relative abundance of Phascolarctobacterium was negatively correlated with the litter weight of piglets at day 21 of lactation ${ }^{15}$ and higher abundance of Phascolarctobacterium was associated with gestational diabetes mellitus in pregnant women (Cortez et al., 2018). Uterine Filifactor, which had higher abundant in the Low group compared to the High group, as showed in the analyses based on NBD and MUM, has been demonstrated to be a predictor of metritis in dairy cows ${ }^{16}$. In addition, there are evidences that Filifactor and Campylobacter are important oral pathogens and are associated with infection-related preterm birth in humans ${ }^{17}$. Staphylococcus was more abundant in the High group in the analyses based on NSB and MUM, contrasting with some finding in the literature which associated high Staphylococcus abundance with lower pregnancy rate in humans ${ }^{18}$. This bacteria genus was identified as a DAM in the analyses of NSB and MUM. Many species of Treponema have been reported to be a pathogenic bacteria ${ }^{19}$. This bacterium was more abundant in the Low group as in the analyses of TNB and Join. To the best of our knowledge, there is no information associated with reproductive performance on Erysipelotrichaceae unclassified, Acinetobacter, and Faecalibacterium. Other bacteria, such as Fusobacterium, Bacteroides, and Lachnospiraceae. Fusobacterium, which was identified to be more abundant in the High group when the groups were defined based on TNB, is a dysbiosis-associated pathogen ${ }^{20}$. Bacteroides and Lachnospiraceae were more abundant in the Low group compared to the High when the groups were defined based on NBD. Bacteroides has been associated with bacterial vaginosis infertility in humans and reproductive disorders in cattle ${ }^{2}$. Lachnospiraceae has been shown to be associated with prediction of pregnancy status ${ }^{2}$ and, similar to Phascolarctobacterium, its abundance was negatively correlated with the litter weight of piglets at day 21 of lactation ${ }^{15}$. In general, there was a higher abundance of noxious bacteria in the Low performance group compared to the High. Interestingly, among these bacteria genera, the abundance of Bacteroides and Phascolarctobacterium in the vaginal microbiome of commercial gilts has been shown to be moderately heritable using the same data presented in this study ${ }^{21}$, suggesting the possibility of performing genetic selection over specific bacteria in the microbiota. For the effect of time, the great majority of the microbes differentially expressed between days had higher relative abundance on D52 compared to D4, including the main microbes that were identified in several analysis: Ruminococcaceae, Clostridium sensu stricto, and Lachnospiraceae. Factors such as age and development of immune defense against the virus after vaccination may be associated with these changes; however, they are completely confounded limiting our conclusion on these findings.

Greater microbiota diversity was observed in the Low group compared to the High group for some of the traits used to define groups. These differences were identified for Fisher, Shannon and Simpson diversities, which account for the total number of species, the proportions of those species, and community evenness. Interestingly, Wang et al. ${ }^{15}$ reported low diversity in the group of sows with low litter performance based on the litter weigh at 21 days of lactation. Therefore, it is possible that a higher abundance of noxious bacteria in the vaginal microbiota of Low performance sows generated the increase in alpha-diversity in this group. On the other hand, in humans, bacterial vaginosis, which decreases fertility, has been associated with greater alpha-diversities ${ }^{22}$.

The LDA revealed that the vaginal microbiota was able to distinguish between Low and High groups of farrowing performance. When using microbiome information from both days, all LDA had correct classification rates of $100 \%$, indicating that the use of OTUs to classify animals with contrasting farrowing performance is very promising. For the analyses performed by day, the worst accuracy of classification $($ AUC $=0.89)$ was obtained 


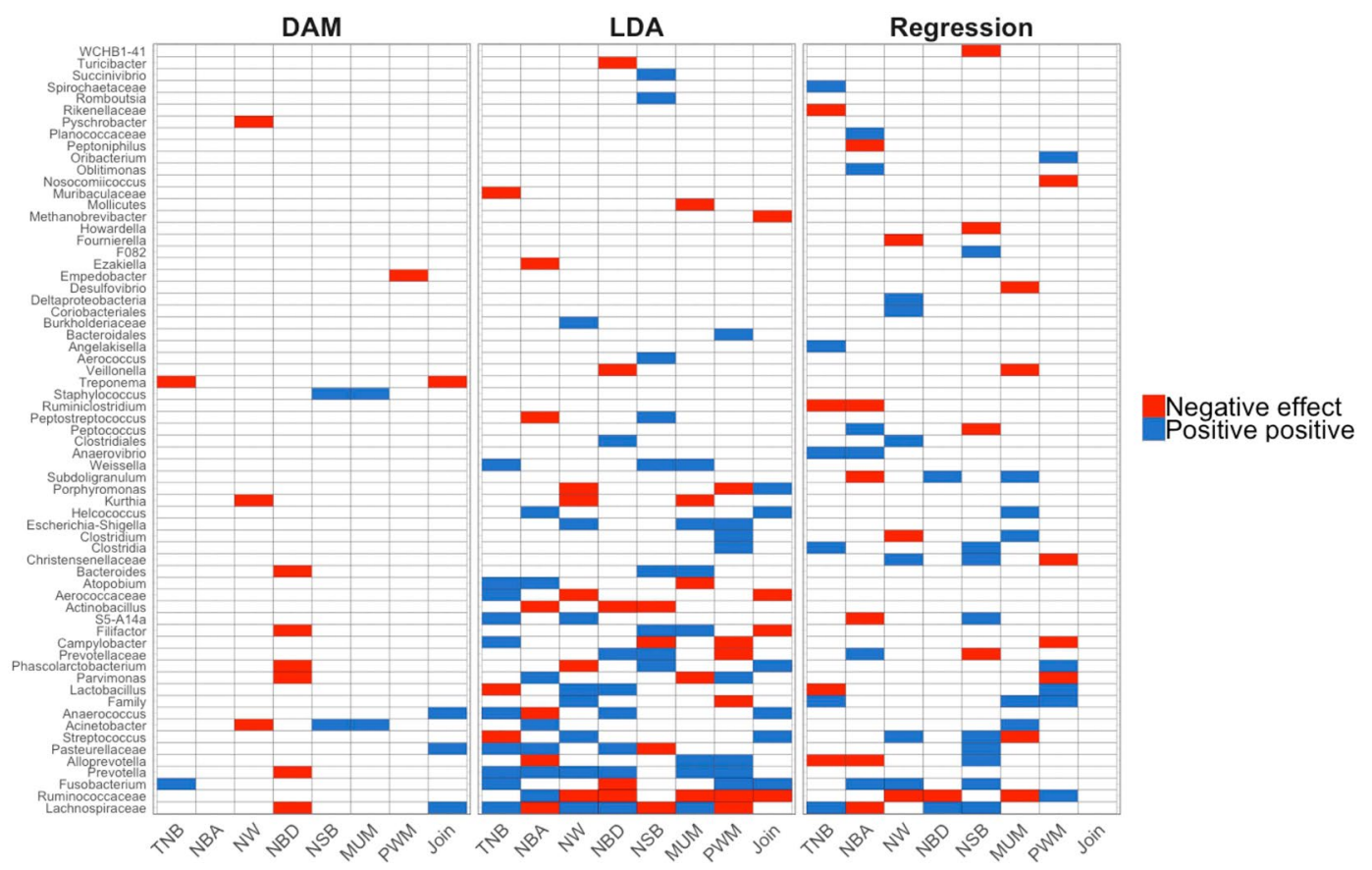

Figure 5. Overall view of the bacteria genera identified for each analysis: differentially abundant microbes (DAM), linear discriminant analyses (LDA), and stepwise regression (Regression). Blue and red represent the positive and negative effects, respectively, of a given bacteria on the farrowing performance: total number born, TNB; number born alive, NBA; number weaned, NW; number of stillborn, NSB; number born mummified, MUM; number born dead, NBD; pre-weaning mortality, PWM; combination of NBA and NBD (Join).

when the groups were based on MUM (using D4 OTU data). This may be due to the fact that the average farrowing performance between the two groups was not very different for this trait (1.1 vs. 0 piglets for the Low and High-performance groups, respectively). In addition to MUM, NBA (using D4 OTU data) and NW (using D52 OTU data), had one misclassified sample (out of 20). In two of these cases (NBA and NW), a sample from the High performance group was incorrectly classified into the Low performance group sample, suggesting that the chance of a gilt with Low performance being classified as High performance is minimal. The bacteria with higher influence on classification into groups in the LDA were very similar to those identified as DAMs in the univariate analyses: Campylobacter, Bacteroides, Porphyromonas, Lachnospiraceae unclassified, Anaerococcus, Filifactor, and Prevotella. This suggests that these bacteria could be used as potential biomarkers between the Low and High performance groups.

The use of vaginal microbiota to classify gilts into farrowing performance groups would enhance the process of culling, allowing the identification of low reproductive producers at an early age prior to breeding. The classification based on the microbiota data from D4 had slightly worse performance than from D52; however, this difference was not large enough to discard the possibility of obtaining the microbiota earlier measures of the vaginal microbiome for diagnostic purposes. Therefore, collection on D4 seems to be promising to identify gilts with better future farrowing performance. In addition, vaginal swabs collected after PRRS virus vaccination shown to be a good tool to obtain the microbiota since it is minimally invasive and can be performed at early ages.

The LDA was effective in identifying additional influential OTUs that were not identified in the univariate analyses (i.e., identification of DAMs). The LDA is a multivariate approach, which allows the relationship within the microbiota to be accounted for, enhancing the understanding behind the association between the microbiota and phenotypes of interest.

The stepwise regression analyses showed that there is a moderate relationship between vaginal microbiota and farrowing performance, with the vaginal microbiota explaining up to $46 \%$ (i.e., NSB) of the variation (i.e., $R^{2}$ ) in subsequent farrowing performance. Lachnospiraceae was identified in most of the analyses (6 in total), corroborating the relationship of this bacteria general with farrowing performance in pigs ${ }^{15}$ (Fig. 5). In addition, Ruminococcaceae, Subdoligranulum, and Alloprevotella were selected in most ( four analyses) of the stepwise regression analyses (Fig. 5). These results were not as promising as those from the LDA. While sows with extreme performance were used for the LDA, all animals were used to perform regression analyses of OTUs on farrowing performance. Hence, in these analyses, we evaluated the impact of OTUs across all values of performance. Therefore, based on these results, although there is potential in identifying contrasting performance with OTU data, the use of these data needs to be further evaluated before they can be used to predict overall farrowing performance.

The vaginal microbiota of commercial replacement gilts was associated with subsequent farrowing performance. In this study we identified major differentially abundant microbes in vaginal samples obtained at the commercial level between gilts that were subsequently classified as having Low and High farrowing performances. 


\begin{tabular}{|l|l|l|l|l|l|l|l|}
\hline & & & & \multirow{2}{*}{} & \multicolumn{2}{|l|}{ Mean (SD) per group } \\
\cline { 6 - 9 } & & & & & \\
Traits $^{\mathbf{a}}$ & $\mathbf{N}^{\mathbf{b}}$ & Mean & SD & Min & Max & Low $(\mathbf{n}=10)$ & High $(\mathbf{n}=10)$ \\
\hline TNB & 96 & 12.4 & 3.1 & 2 & 19 & $6.4(2.5)$ & $17.7(1.3)$ \\
\hline NBA & 96 & 11.6 & 3.2 & 1 & 19 & $4.4(2.3)$ & $16.5(1.4)$ \\
\hline NW & 93 & 11.5 & 1.7 & 5 & 15 & $9.7(0.7)$ & $13.3(0.8)$ \\
\hline NBD & 96 & 0.8 & 1.4 & 0 & 12 & $5.1(2.7)$ & $0.0(0.0)$ \\
\hline NSB & 96 & 0.5 & 0.9 & 0 & 8 & $3.4(1.9)$ & $0.0(0.0)$ \\
\hline MUM & 96 & 0.3 & 0.8 & 0 & 7 & $1.1(1.6)$ & $0.0(0.0)$ \\
\hline PWM & 69 & 2.7 & 1.8 & 1 & 10 & $5.1(2.7)$ & $1.1(0.3)$ \\
\hline
\end{tabular}

Table 1. Descriptive statistics of the farrowing performance data. ${ }^{\mathrm{a} T N B}$, total number born; NBA, number born alive; NW, number weaned; NBD, number born dead; NSB, number of stillborn; MUM, number born mummified; PWM, pre-weaning mortality; ${ }^{b} \mathrm{~N}$ : number of records. ${ }^{c}$ Mean and standard deviation (SD) from Low and High farrowing performance groups.

Gilts with lower subsequent reproductive performance had higher microbiota diversity, suggesting a state of dysbiosis in low-performing animals. High accuracy (100\%) can be obtained by using vaginal microbiome data to classify sows into Low and High farrowing performance groups. In contrast, the vaginal microbiota had limited impact in explaining variation in reproductive performance when the whole data set was used, with $R^{2}$ up to 0.46 for number of stillborn. Particularly, few genera, such as Campylobacter, Bacteroides, Porphyromonas, Lachnospiraceae unclassified, Prevotella, and Phascolarctobacterium were identified as potential biomarkers of farrowing performance in pigs. In conclusion, this study demonstrates that there is potential in using vaginal microbiota data collected after MVL PRRS vaccination to classify sows with Low and High farrowing performance. Future work should focus on validating bacteria associated with farrowing performance identified in this study, evaluating classification of animals with contrasting performance across multiple parities, as well evaluating the use of vaginal microbiota data collected at different time points and without vaccination.

\section{Methods}

All methods described in this study were approved by the Institutional Animal Care and Use Committee at Iowa State University (IACUC\# 6-17-8551-S), following the guidelines and regulations according to the Animal Welfare Act (AWA).

Animals, sample collection, and reproductive performance traits. Ninety-six F1 (Landrace x Large White) replacement gilts from a commercial farm in North Carolina, USA, were vaccinated (133 \pm 11 days old) intramuscularly with a commercial MLV PRRS vaccine (Ingelvac PRRS MLV, Boehringer Ingelheim Animal Health), following the manufacturer's guidelines. Prior to sample collection, the vulva was cleaned to minimize contamination from skin bacteria into the vagina using water and 70\% ethanol. Vaginal swabs were then collected from all gilts using ESwabs (COPAN Diagnostics Inc., Murrieta, CA) at D4 and D52 after vaccination. These 96 animals were selected from a larger pool of 302 sows (described in Sanglard et al. ${ }^{21}$ ) that had first-farrowing performance from January to June 2018 ( 150 days post second microbiome collection). In order to work with a more homogeneous dataset (with regards to the time of farrowing), we identified a narrower period of time (March 2018) that included a larger subset of these 302 animals. Subsequent analyses were done using this subset of 96 animals. These animals had data recorded for NBA, NW, MUM, NSB, and PWM. Number born dead was calculated as the sum of MUM and NSB, and TNB was calculated as the sum of NBA and NBD. Summary statistics of the data are presented in Table 1.

Vaginal microbiota data. The vaginal microbiota data has been previously described in Sanglard et al. ${ }^{21}$. DNA from the vaginal swabs was extracted to profile the vaginal microbiota by $16 \mathrm{~S}$ rRNA gene sequencing. Bacterial DNA was extracted using the Qiagen DNeasy PowerSoil (QIAGEN Inc., Valencia, CA), extraction kit. Briefly, PCR amplicon libraries targeting variable region 4 (V4) of the 16S rRNA gene were produced using a barcoded primer set adapted for Illumina HiSeq. 2000 and MiSeq. ${ }^{23}$. DNA sequence data were generated using Illumina MiSeq paired-end sequencing at the Environmental Sample Preparation and Sequencing Facility (ESPSF) at Argonne National Laboratory (Lemont, IL). Specifically, 515F and 806R primers that included the sequencer adaptor sequences used in the Illumina flow cell were used to PCR amplify the V4 region of the 16S rRNA gene ${ }^{23}$. The $515 \mathrm{~F}$ amplification primer also contained a twelve base barcode sequence that supported pooling and subsequent demultiplexing of up to 2,167 samples in each lane were included ${ }^{23,24}$. Each $25 \mu \mathrm{L}$ PCR reaction contained 9.5 $\mu \mathrm{L}$ of MO BIO PCR Water (Certified DNA-Free), $12.5 \mu \mathrm{L}$ of QuantaBio's AccuStart II PCR ToughMix (2x concentration, $1 \mathrm{x}$ final), $1 \mu \mathrm{L}$ Golay barcode tagged forward primer $(5 \mu \mathrm{M}$ concentration, $200 \mathrm{pM}$ final), $1 \mu \mathrm{L}$ reverse primer $(5 \mu \mathrm{M}$ concentration, $200 \mathrm{pM}$ final), and $1 \mu \mathrm{L}$ of template DNA. The conditions for PCR were as follows: $94^{\circ} \mathrm{C}$ for 3 minutes to denature the DNA, with 35 cycles at $94^{\circ} \mathrm{C}$ for $45 \mathrm{~s}, 50^{\circ} \mathrm{C}$ for $60 \mathrm{~s}$, and $72{ }^{\circ} \mathrm{C}$ for $90 \mathrm{~s}$; with a final extension of $10 \mathrm{~min}$ at $72^{\circ} \mathrm{C}$ to ensure complete amplification. Amplicons were then quantified using PicoGreen (Invitrogen) and a plate reader (Infinite $200 \mathrm{PRO}$, Tecan). Once quantified, volumes of each of the products were pooled into a single tube so that each amplicon was represented in equimolar amounts. This pool was then cleaned up using AMPure XP Beads (Beckman Coulter), and then quantified using a fluorometer (Qubit, Invitrogen). After quantification, the molarity of the pool was determined and diluted down to $2 \mathrm{nM}$, denatured, and then diluted to a final concentration of $6.75 \mathrm{pM}$ with a $10 \%$ PhiX spike for sequencing on the Illumina MiSeq. Amplicons were 
sequenced on a $151 \mathrm{bp}$ MiSeq run using customized sequencing primers and procedures ${ }^{23}$. Mothur was used for sequencing analysis ${ }^{25}$, which followed mothur's MiSeq standard operating protocol. Barcode sequences, primer and low-quality sequences were trimmed using a minimum average quality score of 35 , with a sliding window size of $50 \mathrm{bp}$. Chimeric sequences were removed using Chimera Uchime. For alignment and for taxonomic classification, the SILVA SSU NR reference database $v 132^{26}$ provided by the mothur website was used. The sequences were clustered into OTUs with a cutoff of $99 \% 16 \mathrm{~S}$ rRNA gene similarity. The OTUs were numbered in order of abundance (i.e., OTU1 corresponds to the most abundant OTU). More than 2,000 OTUs were obtained, but after removing OTUs that were absent in more that $10 \%$ of the samples, 1,386 OTUs were used for microbial analyses. For analyses purposes, the relative abundance of the OTUs was calculated by dividing the counts of each OTU by the total number of counts for a given sample. Alpha-diversity was obtained for each day separately, and measured as: Chaol, inverse Simpson, Simpson, Shannon, Fisher, and abundance-based coverage estimator. Analyses of microbiota diversity were performed in $\mathrm{R}^{27}$ using the microbiome package ${ }^{28}$.

Statistical analyses. Identification of animals with contrasting reproductive performance. In order to assess the difference in microbial composition and diversity between sows with contrasting farrowing performance, the sows were split into two farrowing performance groups (Low and High). The phenotypes NW and PWM were pre-adjusted for the number of piglets fostered at birth by adding this in the model. Then, sows were identified as Low (bottom 10) or High (top 10) performers after being ranked based on each of these traits separately: NBA, NBD, TNB, NW, MUM, NSB, PWM, and a combination of high farrowing performance for NBA and NBD (Join). Join was created by double-sorting the sows by the NBA and, then, by the NBD in opposite directions to obtain groups with high and low farrowing outcomes. Since microbiota data were collected at D4 and D52, a total of 40 samples (20 animals with two samples representing each time point) were used in these analyses (combination between farrowing performance group and collection day). The average farrowing performance of each group is given in Table 1.

Identification of differentially abundant microbes (DAM), and assessment of microbial alpha-diversity between sows with Low and High farrowing performance. For the identification of DAMs, the following repeated measurements negative binomial mixed model was used:

$$
y_{i j k}=\mu+\operatorname{group}_{i}+\text { day }_{j}+\left(\text { group } * d a y_{i j}+\beta_{1} \operatorname{age}_{k}+\operatorname{animal}_{k}+\log \left(L_{i j k}\right)\right.
$$

where $y_{i j k}$ is the raw count for the OTU analyzed; $\mu$ is the overall mean; group $p_{i}$ is the fixed effect of the $i^{\text {th }}$ farrowing performance group (Low or High); day is the fixed effect of the $j^{\text {th }}$ day of collection (D4 or D52); $\beta_{1}$ is the partial regression coefficient for the covariate age at collection for the $k^{\text {th }}$ animal $\left(\right.$ age $\left._{k}\right)$; animal ${ }_{k}$ is the random effect of the $k^{\text {th }}$ animal, assuming animal $\sim N\left(0, I \sigma_{\text {animal }}^{2}\right)$, where $\boldsymbol{I}$ is the identity matrix; and $\log \left(L_{i j k}\right)$ is the TMM-normalized library size, used as an offset. The TMM normalization ${ }^{29}$ factors used to normalize library size were obtained based on all the OTUs in the dataset $(\sim 2,000)$ and all animals that had vaginal microbiota data collected ( $\sim 300$; data not shown). A false-discovery rate correction was applied for multiple testing $\operatorname{correction}^{30}$, and DAMs for farrowing performance groups were identified when $q$-value $<0.05$. Results are being presented as $\log _{2}$ fold change ( $\log _{2} \mathrm{FC}$ ) of the High group compared to the Low (i.e., positive numbers correspond to greater abundance in the High group).

For alpha-diversity, a linear mixed model including the same effects in Eq. 1 was used, with the exception that the offset was removed from the model. The Shapiro-Wilk test for normality was applied and, when the normality assumption of the residuals was not met $(P$-value $<0.05)$, the response data was log-transformed ${ }^{31}$. Analyses were performed using the GLIMMIX procedure of SAS 9.4 (Statistical Analysis System; Cary, NC, USA), and the calculation of $q$-values were done with the p.adjust function from the stats package in $\mathrm{R}$.

Classification of farrowing performance based on OTU relative abundance. Linear discriminant analyses were performed using the OTU data to classify animals into the two farrowing performance groups (i.e., Low and High groups). For the analyses, microbiome information from D4 and D52 were used to classify into farrowing groups. In addition, analyses were done separately for D4 and D52 in order to optimize the classification on animals.

Prior to LDA, the relative abundance of each OTU was calculated as the proportion of a given OTU divided by the library size for each animal and, then, pre-adjusted for the fixed effect of the covariate age. Next, a stepwise linear discriminant analysis was performed over the adjusted phenotype in order to identify significant $(P$-value $<0.05)$ OTUs to be included in the discriminant model using stepwise selection. After selection of OTUs, a leave-one-out cross-validation was used to assess the predictive ability of the OTUs to correctly classify samples into the correct groups (Low or High). A ROC curve was used to assess the accuracy (i.e., AUC) of the binary classifier diagnostic. The ROC curve was created by plotting the true positive rate (sensitivity) against the false positive rate ( 1 - specificity) at various threshold settings. LDA were performed using the STEPDISC and DISCRIM procedures of SAS 9.4. The AUC was calculated with $p R O C$ package ${ }^{32}$ from $\mathrm{R}$.

Prediction of farrowing performance based on OTU. A stepwise linear regression of OTUs on reproductive traits was performed to assess the overall relationship between OTU abundance and farrowing performance. Prior to analyses, the data were pre-adjusted to remove systematic effects. In this step, the reproductive data were adjusted for the fixed effects of farrowing contemporary group (combination of month/year of farrow and farm) and the relative abundance OTU data were adjusted for the covariate of age at microbiota collection. Afterwards, the pre-adjusted reproductive data were used as response variable, one at a time, and the pre-adjusted data of all OTUs were used as explanatory variables. The OTUs were selected to enter and remain in the model in a stepwise approach, using a threshold of $P$-value $<0.15$. Analyses were performed using the GLMSELECT procedure of SAS 9.4. 
Received: 24 October 2019; Accepted: 3 February 2020;

Published online: 20 February 2020

\section{References}

1. Llamas-López, P. J., López-Úbeda, R., López, G., Antinoja, E. \& García-Vázquez, F. A. A new device for deep cervical artificial insemination in gilts reduces the number of sperm per dose without impairing final reproductive performance. J. Anim. Sci. Biotechnol. 10, 1-9 (2019).

2. Mcclure, M. W. The Vaginal Microbiome Related to Reproductive Traits in Beef Heifers, Master's thesis. ScholarWorks (University of Arkansas, 2018).

3. Cho, I. \& Blaser, M. J. The human microbiome: at the interface of health and disease. Nat. Rev. Genet. 13, 260-70 (2012).

4. Bicalho, M. L. S. et al. Dynamics of the microbiota found in the vaginas of dairy cows during the transition period: Associations with uterine diseases and reproductive outcome. J. Dairy Sci. 100, 3043-3058 (2017).

5. Borgogna, J. L. C. \& Yeoman, C. J. The Application of Molecular Methods Towards an Understanding of the Role of the Vaginal Microbiome in Health and Disease. Methods in Microbiology 44, (Elsevier Ltd., 2017).

6. Kuczynski, J. et al. Experimental and analytical tools for studying the human microbiome. Nat. Rev. Genet. 13, 47-58 (2012).

7. Serão, N. V. L. et al. Genetic analysis of reproductive traits and antibody response in a PRRS outbreak herd. J. Anim. Sci. 92, 2905-2921 (2014).

8. Putz, A. M. et al. The effect of a porcine reproductive and respiratory syndrome outbreak on genetic parameters and reaction norms for reproductive performance in pigs. J. Anim. Sci. 97, 1101-1116 (2019).

9. Lopez, O. J. \& Osorio, F. A. Role of neutralizing antibodies in PRRSV protective immunity. Vet. Immunol. Immunopathol. 102, 155-163 (2004).

10. Balasch, M. et al. Immune response development after vaccination of 1-day-old naïve pigs with a Porcine Reproductive and Respiratory Syndrome 1-based modified live virus vaccine. Porc. Heal. Manag. 5, 1-13 (2019).

11. Niederwerder, M. C. Role of the microbiome in swine respiratory disease. Vet. Microbiol. 209, 97-106 (2017).

12. Neuman, H., Debelius, J. W., Knight, R. \& Koren, O. Microbial endocrinology: The interplay between the microbiota and the endocrine system. FEMS Microbiol. Rev. 39, 509-521 (2015).

13. Queipo-Ortuño, M. I. et al. Gut Microbiota Composition in Male Rat Models under Different Nutritional Status and Physical Activity and Its Association with Serum Leptin and Ghrelin Levels. PLoS One 8 (2013).

14. Vlčková, K. et al. Impact of stress on the gut microbiome of free-ranging western lowland gorillas. Microbiol. (United Kingdom) 164, 40-44 (2018).

15. Wang, H. et al. Differential analysis of gut microbiota correlated with oxidative stress in sows with high or low litter performance during lactation. Front. Microbiol. 9, 1-11 (2018).

16. Jeon, S. J. et al. Uterine microbiota from calving until establishment of metritis in dairy cows. Soo. J. Anim. Sci. 93 (2019).

17. Cobb, C. M. et al. The oral microbiome and adverse pregnancy outcomes. Int. J. Womens. Health 9, 551-559 (2017).

18. Franasiak, J. M. \& Scott, R. T. Reproductive tract microbiome in assisted reproductive technologies. Fertil. Steril. 104, 1364-1371 (2015).

19. Stamm, L. V., Walker, R. L. \& Read, D. H. Genetic diversity of bovine ulcerative mammary dermatitis-associated Treponema. Vet. Microbiol. 136, 192-196 (2009).

20. Wu, N. et al. Dysbiosis Signature of Fecal Microbiota in Colorectal Cancer Patients. Microb. Ecol. 66, 462-470 (2013).

21. Sanglard, L. P. et al. Investigating the relationship between vaginal microbiota and host-genetics and their impact on immune response and farrowing traits in commercial gilts. J. Anim. Breed. Genet. 137, 84-102 (2019).

22. Schachtschneider, K. M. et al. Modulation of Systemic Immune Responses through Commensal Gastrointestinal Microbiota. PLoS One 8 (2013).

23. Caporaso, J. G. et al. Ultra-high-throughput microbial community analysis on the Illumina HiSeq and MiSeq platforms. ISME J. 6, 1621-1624 (2012).

24. Caporaso, J. G. et al. Global patterns of $16 \mathrm{~S}$ rRNA diversity at a depth of millions of sequences per sample. Proc. Natl. Acad. Sci. USA 108(Suppl), 4516-22 (2011).

25. Kozich, J. J., Westcott, S. L., Baxter, N. T., Highlander, S. K. \& Schloss, P. D. Development of a Dual-Index Sequencing Strategy and Curation Pipeline for Analyzing Amplicon Sequence Data on the MiSeq Illumina Sequencing Platform. Appl. Environ. Microbiol. 79, 5112-5120 (2013).

26. Quast, C. et al. The SILVA ribosomal RNA gene database project: Improved data processing and web-based tools. Nucleic Acids Res. 41, 590-596 (2013).

27. R Development Core Team. R: A language and environment for statistical computing. R Foundation for Statistical Computing, Vienna, Austria, ISBN 3-900051-07-0 (2008).

28. Lahti, L. \& Shetty, S. Tools for microbiome analysis in R. (2017).

29. Robinson, M. D., Oshlack, A. \& others A scaling normalization method for differential expression analysis of RNA-seq data. Genome Biol. 11, R25 (2010).

30. Storey, J. A direct approach to false discovery rates. J. R. Stat. Soc. B. 64, 479-498 (2002).

31. Royston, J. P. Some Techniques for Assessing Multivarate Normality Based on the Shapiro-Wilk. R. Stat. Soc. 32, 121-133 (1983).

32. Robin, X. et al. pROC: an open-source package for R and S+ to analyze and compare ROC curves. BMC Bioinformatics 8, 12-77 (2011).

\section{Acknowledgements}

The authors thank the financial support of the Iowa Pork Producers Association and the USDA-NRSP-8 Swine Genome Coordination Effort, and the in-kind support of Smithfield Premium Genetics.

\section{Author contributions}

L.P.S.: performed data analyses, interpreted results, and drafted manuscript; K.A.G., J.C.M.D. and N.V.L.S. developed the research project. L.P.S., J.C.M.D. and N.V.L.S. conceived statistical analyses. L.P.S. and N.V.L.S. prepared a first draft of the manuscript and all authors contributed to the final manuscript. S.S.E. and C.J.Y. performed the microbiome sequencing analysis. K.A.G. coordinated the collection of materials. D.C.L. performed the collection of blood samples. M.C.N. and D.C.L. performed the antibody measurement analysis. All authors read and approved the final manuscript.

\section{Competing interests}

The authors declare no competing interests. 


\section{Additional information}

Supplementary information is available for this paper at https://doi.org/10.1038/s41598-020-59955-8.

Correspondence and requests for materials should be addressed to N.V.L.S.

Reprints and permissions information is available at www.nature.com/reprints.

Publisher's note Springer Nature remains neutral with regard to jurisdictional claims in published maps and institutional affiliations.

(c) (i) Open Access This article is licensed under a Creative Commons Attribution 4.0 International License, which permits use, sharing, adaptation, distribution and reproduction in any medium or format, as long as you give appropriate credit to the original author(s) and the source, provide a link to the Creative Commons license, and indicate if changes were made. The images or other third party material in this article are included in the article's Creative Commons license, unless indicated otherwise in a credit line to the material. If material is not included in the article's Creative Commons license and your intended use is not permitted by statutory regulation or exceeds the permitted use, you will need to obtain permission directly from the copyright holder. To view a copy of this license, visit http://creativecommons.org/licenses/by/4.0/.

(c) The Author(s) 2020 